\title{
Experience of Using OWL Ontologies for Automated Inference of Routine Pre-operative Screening Tests
}

\author{
Matt-Mouley Bouamrane ${ }^{1}$, Alan Rector ${ }^{2}$, and Martin Hurrell ${ }^{3}$ \\ 1 College of Medical, Veterinary and Life Sciences \\ Centre for Population and Health Sciences \\ University of Glasgow, Scotland, U.K. \\ Matt-Mouley.Bouamrane@glasgow.ac.uk \\ 2 School of Computer Science, Manchester University, U.K. \\ Rector@cs.man.ac.uk \\ 3 CIS Informatics, Glasgow, Scotland, U.K. \\ martin.hurrell@informatics.co.uk
}

\begin{abstract}
We describe our experience of designing and implementing a knowledge-based pre-operative assessment decision support system. We developed the system using semantic web technology, including modular ontologies developed in the OWL Web Ontology Language, the OWL Java Application Programming Interface and an automated logic reasoner. Using ontologies at the core of the system's architecture permits to efficiently manage a vast repository of pre-operative assessment domain knowledge, including classification of surgical procedures, classification of morbidities, and guidelines for routine pre-operative screening tests. Logical inference on the domain knowledge, according to individual patient's medical context (medical history combined with planned surgical procedure) enables to generate personalised patients' reports, consisting of a risk assessment and clinical recommendations, including relevant pre-operative screening tests.
\end{abstract}

\section{Introduction}

In the U.K., a typical patient pathway to surgery involves the following steps: referral from primary care to an outpatient clinic in a hospital, pre-operative assessment of the patient at the hospital, the actual surgery, discharge from hospital and a return to community-based care. Pre-operative assessment is a routine medical evaluation and screening process which takes place prior to surgery in order to assess a patient's fitness for surgery, while identifying potential risk factors. Pre-operative screening is designed as an opportunity for taking appropriate action which can be beneficial to the patient. Screening is an opportunity to alter the clinical management of a patient scheduled for elective surgery, as new information come to the attention of health professionals. This information may warrant additional specific precautions, such as making provisions for additional resources (e.g. requesting some specialist equipment or booking a bed 
in an Intensive Care Unit), requesting a specific intervention (e.g. pre-operative treatment or intervention, referral to specialist consultant) or even cancelling the planned surgery. Thus, the period between pre-operative assessment and surgery is seen as an important opportunity to anticipate risks and optimise the patient fitness for surgery. At this stage, routine ordering of pre-operative screening tests has long been a common practice within hospitals. Pre-operative tests may be requested both for asymptomatic patients and patients with specific risk factors (e.g. patients with a history of cardio-vascular or respiratory co-morbidities).

We describe our experience of developing a knowledge-based decision support system designed to assist health professionals in secondary care during the pre-operative assessment of patient prior to elective surgery. We review related work on medical decision support systems and background information on the pre-operative assessment process in section 2. We discuss design features of the system, including technology and specificity of the domain knowledge in section 3. We describe the iterative development of the system in section 4. We discuss our experience of implementing pre-operative test guidelines in section 5 and conclude with some final remarks and future work.

\section{Pre-operative Assessment and Decision Support Systems}

\subsection{Medical Computer Decision Support Systems}

The majority of errors within health delivery systems are not necessarily due to human errors, but are rather often consequences of broader systemic flaws in the organisation of processes and services 1]. The potential benefits of integrating computer-based Clinical Decision Support Systems (CDSS) 23456778910 within work practices include the ability to:

- (i) influence clinicians behaviour and reduce variability of outcomes across various health professionals and increase the standardisation of processes towards evidence-based guidelines.

- (ii) combine and synthesise complex related pieces of information.

- (iii) facilitate access to clinical information and reporting of results through greater accessibility of data and improved display of information (e.g. using graphs, charts...)

- (iv) support the generation of patient-specific (medical history) and context specific (e.g. according to morbidity, surgical intervention, local hospital rules, etc.) prompts and reminders.

- (v) reduce medication adverse events with computer assisted order entry through a reduction of misread manual writing, notifications of adverse interaction, allergies, etc.

- (vi) identify patterns within the patient data which must be acted upon (e.g. abnormal or inconsistent findings, alerts, ordering of tests and further investigations, referral to specialist consultant...) 
- (vii) doing all of the above while preserving health professionals' independence and ability to tailor patient care according to individual circumstances, specific needs, availability of resources or other constraints.

If successfully embedded within routine work practices CDSSs can become important process standardisation and error preventing tools. While CDSSs have generally proved reliable whenever rules and guidelines are clearly applicable, their record on emulating medical diagnosis is rather less obvious. This is due to the inherent difficulty and complexity in designing explicit conceptual models of the medical diagnosis thought process, except perhaps in the most straightforward cases, which would limit the usefulness of such systems.

CDSSs have other inherent limitations: recommendations issued by the systems can only be as good as the guidelines they model, and as a result, flaws in the guidelines will unfortunately be systematically reproduced in the output of the system [6]. Clinical knowledge is always limited or partial and it is not unusual for certain guidelines to be revised or proved wrong. Ironically, in these situations, patients' health would actually benefit from errors of omissions which a CDSS would make less likely [7. There is obviously little CDSS designers can do about this problem apart from updating the system whenever new guidelines are introduced or old ones revised.

Several systematic reviews [2 3448910] concluded that CDSSs had generally (although not always...) demonstrated some benefits on clinical behaviour, compliance and performance during clinical controlled trials, including drug dosing and prescribing systems, preventive care and other generic or disease-specific systems. Regarding patients outcomes, results are less clear cut, in part due to the sparsity of available studies in that respect. In contrast, these studies showed that diagnostic aids have generally demonstrated little benefit to clinical practice overall (see Taylor's insightful description of the issues surrounding health informatics for a potential explanation of the lack of successes of diagnostic aids in [1]]).

\subsection{Pre-operative Assessment}

In the United Kingdom, a patient due to undergo surgery will typically be referred to a hospital by his family doctor and will then first get an appointment at an out-patient clinic at the hospital for initial screening. The patient will then undergo a pre-operative assessment consisting of: answering a clinical questionnaire, generally followed by a physical examination, certain laboratory tests and possibly referral to a specialist consultant. Patient screening can be performed in a variety of settings: face to face consultations, paper-based questionnaires, on the telephone, or through web-based forms on the internet.

García-Miguel et al. define pre-operative assessment as "the clinical investigation that precedes anaesthesia for surgical or non-surgical procedures, and is the responsibility of the anaesthetist" [12. The primary goal of pre-operative assessment is to maximise a patient's fitness for a (i.e. surgical) procedure by: 
- (i) ensuring that the patient is fully informed about the procedure and has provided informed consent.

- (ii) identifying early in the patient's health pathway potential risks of perioperative (i.e. during) and postoperative complications due to pre-existing conditions (e.g. cardio-vascular or respiratory conditions, chronic diseases, multiple co-morbidities, previous adverse events, etc.).

- (iii) requesting additional investigations (e.g. tests) or referral to a specialist.

- (iv) taking steps to improve patient fitness (e.g. referral to family doctor for smoking cessation, weight loss, chronic disease control and management, etc.)

- (v) allocating appropriate resources for the day of surgery (e.g. taking appropriate actions to deal with patient's allergies, booking specialist equipment or a bed in critical care unit, etc.).

- (vii) considering alternatives to surgery when the risks of surgery are considered too high for the patient's safety.

- (vi) reducing the overall risk of late surgery cancellation by ensuring that all feasible precautionary steps have been taken prior to surgery.

Due to the vast scope of pre-operative assessment, the clinical domain knowledge potentially relevant for assessment is virtually limitless. For this reason, a generic PA has traditionally focused on identifying common allergies, cardiovascular and respiratory risks and pre-empting potential airway complications, such as difficult intubation during anaesthesia. Complex surgical procedures may require additional precautions or even have separate specific pre-operative protocols.

\section{A Knowledge-Base Pre-operative Assessment System}

\subsection{System Design Considerations}

In addition to general considerations relevant to all CDSSs reviewed in the previous section, this research project had several important specific requirements. During the design phase of the project, requirements identified included that the system:

- (i) be capable of capturing highly specific patient medical information in a structured and coherent, yet flexible (i.e. adaptive) manner.

- (ii) have the ability to use and combine heterogeneous sources of clinical information.

- (iii) be capable of making useful inferences based on available evidence-based pre-operative assessment medical knowledge.

- (iv) provide context specific explanations for these medical inferences, targeted at a variety of health professionals (i.e. nurse, doctor, anaesthetist).

- (v) provide some level of transparency regarding the mechanisms for reaching previous medical inferences.

- (vi) provide some mechanisms to conveniently update and maintain the system in the face of new requirements and advances in the availability of evidencebased medical knowledge. 
- (vii) being compatible with earlier versions of the system, including handling data from legacy patient databases while providing the same level of decision support.

\subsection{System Overview}

Figure 1 gives an overview of the general principles behind the architecture of our knowledge-based pre-operative decision support system. The system is composed of five main elements:

- 1. A patient pre-operative medical history information collection module. This component can be designed to be adaptive to the medical context of the information collected for all new patients entered in the system (case 1.a.). The adaptive behaviour of the system is obtained by modelling medical relationships and dependencies in a questionnaire ontology [1314]. For patients whose medical history is already stored in some legacy clinical databases (case 1.b.), the automatic generation of a medical history is obtained through a reverse-engineering mapping from the legacy database information model to the questionnaire ontology [15].

- 2. The previous steps results in the generation of a patient pre-operative medical history representation in OWL [16. There is an important distinction to be

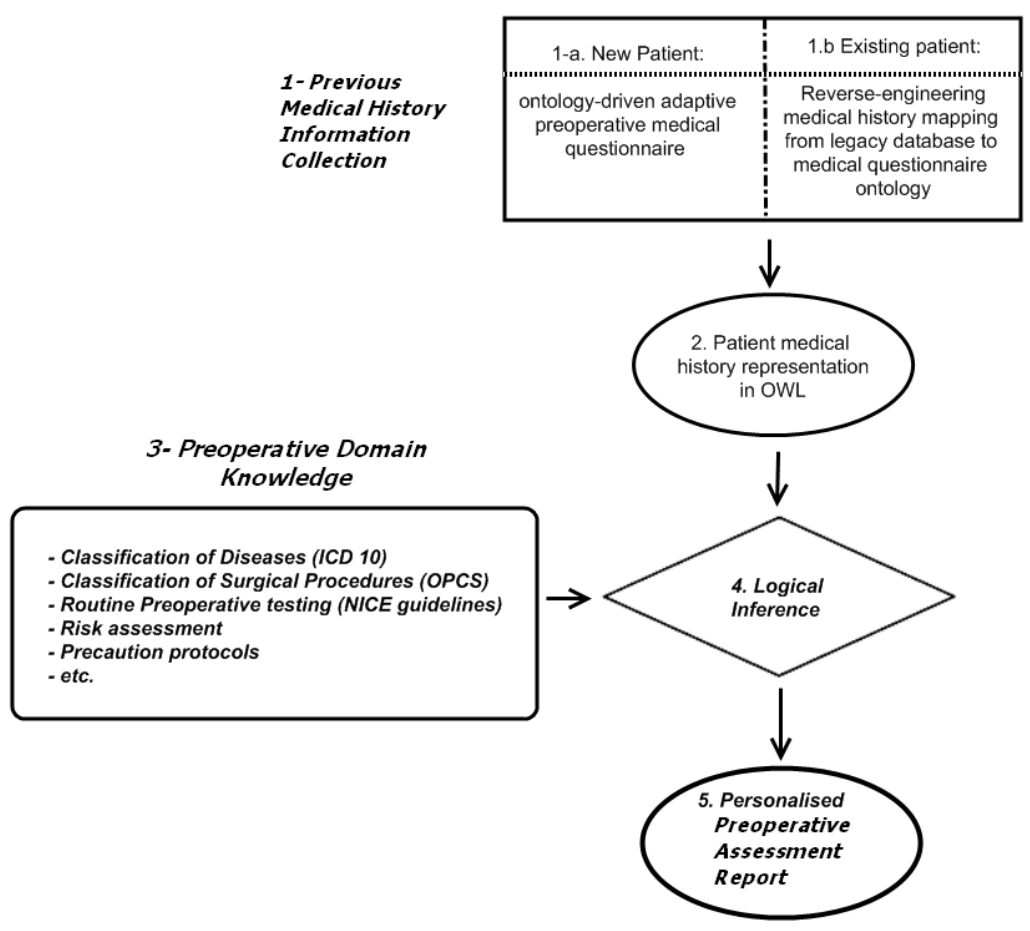

Fig. 1. Overview of Knowledge-based Pre-operative Decision Support System 
made regarding this patient history: some of the information will have be obtained from clinical sources (e.g. examination by nurse or doctor, pre-operative tests, etc.) However, the information directly collected from the patient himself through the patient questionnaire is likely to be "coarse-grained", even if responding to the questionnaire is supervised by a pre-operative nurse, as is usually the case. Consider the following examples to illustrate this last point: a patient may know that he has "diabetes" but may be unable to qualify his condition any further than this. He may know that he is taking medication for a "heart condition" but may not be able to recall the exact name or the type of medication. While this unfortunately places some limitations on the accuracy and reliability of the patient's medical history, this is a consequence of the preoperative process itself rather then a design flaw of the decision support system. What the system can do however is highlight which information was obtained through a reliable clinical source or was obtained from the patient himself, so a health professional can decide to make further investigation on a specific piece of information if necessary or relevant. This additional demand on the workload of the health professional could be somehow alleviated by obtaining the relevant information directly from the medical record at the patient's family doctor if available 1 .

- 3. Because of the nature of pre-operative assessment in attempting to identify relevant risks of complications, the domain knowledge is potentially limitless. However, for practical reasons, pre-operative assessment has generally focused on generic risks (e.g. cardio-vascular, respiratory) unless the patient or surgical procedure require specific attention. We focused on designing a generic pre-operative assessment decision support ontology, including information on classification of morbidities using the ICD-10 International Classification of Diseases, classification of surgical procedures based on OPCS (Office of Population Censuses and Surveys) 2 and other relevant evidence-based pre-operative assessment medical knowledge, such as the NHS National Institute for Clinical Excellence (NICE) routine pre-operative tests guideline 3 . The pre-operative assessment ontology was developed in OWL along the principles of modularity developed by Rector et al. [1718, for coherent and efficient knowledge update and management [19].

- 4. The personalised information representation obtained at step 2 is combined with the general domain knowledge of step 3 in order to make relevant logical inferences on this specific patient [20].

- 5. A personalised patient pre-operative report is compiled including (i) suggested pre-operative tests, (ii) risk assessments and (iii) suggested precaution protocols if relevant to the patient specific medical context.

\footnotetext{
${ }^{1}$ This is the subject of a research fellowship funded by the the Scottish Health Executive, Chief Scientist Office.

2 http://www.connectingforhealth.nhs.uk/systemsandservices/data/ clinicalcoding

3 National Health Service, National Institute for Clinical Excellence http://www.nice.org.uk/Guidance/CG3
} 


\section{System Implementation}

\subsection{System Development Tools}

Prior to introducing semantic technology within the system, the pre-operative software was only composed of the following elements: user input, clinical data storage and a rule engine. The pre-operative risk assessment was then almost entirely based on the calculation of numeric scores. The introduction of semantic based technology in the system enabled adaptive information collection, high level semantic patient modelling and decision support based on patient classification rather than numeric rules only. This provides for a significant enhancement to the functionalities and capabilities of the system. Protégé-OWL was used as the main ontology development tool 21] and we used the web ontology language OWL 22, the java OWL application programming interface 23] and the Pellet reasoner [24].

\subsection{Adaptive Medical Questionnaire}

Context-sensitive adaptation is used to iteratively capture finer-grained information with each successive step, should this information be relevant according to a questionnaire ontology. The proposed method intends to replicate the investigating behaviour exhibited by clinicians when presented with items of information which may be cause for concern or require further attention. While the system has the potential to reduce the number of questions and thus save time and costs for healthy patients, the emphasis is rather on collecting more information whenever relevant so a proper informed patient risk assessment can be performed. The method is robust, scalable and highly configurable [13 14|16].

\subsection{Medical Domain Knowledge of Pre-operative Assessment}

As previously suggested, due to the nature of pre-operative assessment, the clinical domain knowledge relevant for assessment is potentially limitless. Some of the important knowledge resources introduced in the system included:

- Access to a knowledge base of approximately 1700 OPCS classification of surgical procedures. OPCS is the official classification of surgical procedures used by the NHS and is among other things used by hospital trusts to get reimbursed by the NHS for the procedures they carry out in the hospitals throughout the year (i.e. through the Payment by Results, PbR scheme by which funds are allocated according to levels of activity within the trusts). This feature is therefore of critical importance for integration of the application within hospital administrative information management systems. In addition, the OPCS knowledge base include unique OPCS code identifiers, detailed English clinical descriptions of surgical procedures, classification according to 16 major anatomical categories of procedures (e.g. vascular, thorax, abdomen, brain, etc.) and approximately 150 subcategories, allowing for fine grain classification of procedures. The repository 
also provides an overall surgery risk from grade 1 (minor) to grade 4 (major+) for each procedures.

- Access to the International Classification of Diseases, ICD-10 codes. This is a major feature in the application ability to classify elements of patient medical history into defined categories of morbidities (e.g. cardio-vascular, respiratory, renal diseases, unusual symptoms, etc.)

- Both of these previous features are critical to the integration of national and international pre-operative guidelines. We integrated the NICE pre-operative guideline, which is used to determine appropriate screening test investigations for a given patient, based on his individual medical context (medical history and planned surgical procedure). The guidelines are complex and are often not used in practice because healthcare professionals have neither the time nor the knowledge to apply them (see section 5).

\subsection{Mechanism of Decision Support}

In the system, decision support is usually provided in a 2 step process. The first step typically calculates risk scores using numerical formulas such as the Goldman and Detsky cardiac risk index 25]. or derives risk grades (e.g. ASA physical status classification grades 4 . The system does not use the decision support ontology at this stage but merely computes numeric values using an open source Java-based rule engine (JBoss Rules5). Once the risk grades and categories have been derived from the first risk calculation step, the system then performs decision support using the open-source java-based Pellet reasoner to reason on the decision support ontology given a patient OWL medical history profile. Examples of reasoning with the decision support ontology can be found in the next section as well as in 1920].

\section{Pre-operative Tests Recommendations and Reasoning}

\subsection{NICE Pre-operative Tests Guidelines}

In the U.K., the effectiveness of pre-operative screening has been identified as a research priority by the National Institute for Health Research (NIHR) Health Technology Assessment (HTA) programme. A systematic review on preoperative screening tests was commissioned by HTA and published by Munro et al. in 1997 26]. Following up on the previous work, the National Institute for Clinical Excellence (NICE) published in 2003 guideline recommendations on the use of routine pre-operative screening tests. These studies had important implications as they highlighted that: (i) the available evidence on the clinical benefits of routine pre-operative testing was mixed and the quality of studies was

\footnotetext{
${ }^{4}$ (American Society of Anaesthesiologists) ranging from ASA I (healthy patient) to ASA V (moribund).

5 http://www.jboss.com/products/rules

6 NIHR HTA, http://www.hta.ac.uk/
} 
(1) (2)-GRADE IV SURGERY

\begin{tabular}{|c|c|c|c|c|c|c|c|c|c|c|c|c|}
\hline $\begin{array}{l}\text { (3) } 4 \text { COMORBIDITY } \\
\text { COMO }\end{array}$ & & $\begin{array}{r}\text { ASA } \\
\text { Respi } \\
\text { omor }\end{array}$ & $\begin{array}{l}=2- \\
\text { ratory } \\
\text { bidity }\end{array}$ & (4) & $+\mathrm{car}$ & $\begin{aligned} & \text { ASA } \\
& \text { dio-va } \\
& \text { comor }\end{aligned}$ & $\begin{array}{l}=3 \\
\text { iscula } \\
\text { bidity }\end{array}$ & (3) & & $\begin{array}{r}\text { ASA } \\
\text { Ren } \\
\text { Como } \\
\end{array}$ & $\begin{array}{l}=3 \\
\text { al } \\
\text { bidity }\end{array}$ & (3) \\
\hline | Preoperative & & GE in & years & (5) & & GE in & years & (5). & & GE in & years & (5) \\
\hline tests & $16-40$ & $40-60$ & $60-80$ & $80+$ & $16-40$ & $40-60$ & $60-80$ & $80^{\prime}+$ & $16-40$ & $40-60$ & $60-80$ & $80+$ \\
\hline Chest X-ray & & & & & & & YES & YES & & & & \\
\hline ECG & & & YES & YES & YES & YES & YES & YES & & YES & YES & YES \\
\hline Full Blood Count & YES & YES & YES & YES & YES & YES & YES & YES & YES & YES & YES & YES \\
\hline $\begin{array}{l}\text { Haemostasis } \\
\text { Renal function }\end{array}$ & YES & YES & YES & YES & YES & YES & YES & YES & YES & YES & YES & YES \\
\hline Random glucose & NO & NO & NO & NO & NO & NO & NO & NO & & & & \\
\hline Urine analysis & & & & & & & & & & & & \\
\hline & & & & & & & & & & & & \\
\hline Lung function & & & & & NO & NO & NO & NO & NO & NO & NO & NO \\
\hline
\end{tabular}

Fig. 2. NICE pre-operative guidelines. Investigations are recommended based on: (1) type and (2) risk grades of surgical procedure, (3) severity and (4) nature of co-morbidities and (5) patient age. There are 3 types of result for each test: "recommended", "not recommended" and "consider" (in amber).

weak overall and that (ii) there was no evidence to support the use of systematic routine pre-operative screening tests. In order to address the latest point, NICE set up a Guideline Development Group (GDG) to clarify the medical context in which the use of routine pre-operative tests was appropriate and justified by the available clinical evidence. After a thorough 13-steps guideline development process, including 2 parallel expert panels and a wide consultation exercise, NICE issued explicit guidelines for routine pre-operative testing. The guidelines made 3 types of recommendations: (i) test is "recommended", (ii) test is "not recommended" and (iii) "consider" the test based on 5 distinct input: (1) type of surgery (e.g. cardiac surgery, neuro-surgery or non-cardiac surgery) and (2) risk grades of surgical procedures, (3) severity (ASA grade) and (4) nature of comorbidities (cardio-vascular, respiratory or renal) and (5) patient age. A sample of the guidelines is illustrated in Figure 2. The sample illustrates recommendations for various morbidities and severity levels. The guidelines were themselves summarised into various categories of surgery, surgery severity, morbidity categories and morbidity severity 7 .

- Type of investigations: the guidelines include 9 potential investigations: Chest X-Ray, ECG (Electrocardiogram), Full Blood Count, Haemostasis, Renal Function, Random glucose, Urine analysis, Blood gases and Lung Function.

- Type of recommendations: there are currently 3 types of recommendations for each test: "test recommended", "test not recommended" and "consider test".

- Factors Influencing recommendations: There are 5 factors taken into consideration in order to find the relevant recommendations: the (i) age of the patient, (ii) his ASA grade, (iii) the type of co-morbidities the patient has (e.g. respiratory,

7 http://www.nice.org.uk/nicemedia/live/10920/29090/29090.pdf 
cardio-vascular, renal) (iv) the type of surgery (e.g. cardio-vascular surgery, neurosurgery, etc.) (v) the risk grade of the surgery (from 1 to 4 ).

- Number of cases in the guidelines: the guidelines are summarised for preoperative health assessors into 36 tables such as the one illustrated in Figure 2. There are different tables for different combinations of the 5 factors previously described, including different tables for children under 16 years old and adults over 16 years old. In total, there are at least 1242 different possible cases.

As the example in Figure 2 suggests, the guidelines recommend a chest X-Ray test for patients over 60 years old if : they are scheduled for a surgical procedure of severity risk grade IV and if they have a cardio-vascular co-morbidity of severity level $\mathrm{ASA}=3$. An ECG would be recommended for someone aged less than 40 years old if they have a cardio-vascular co-morbidity of level ASA=3 but not necessarily if they have a renal co-morbidity of level $\mathrm{ASA}=3$ or a respiratory co-morbidity of level $\mathrm{ASA}=2$. If the patient is between 40 and 60 years old, an ECG would be recommended if the patient has either a cardio-vascular or a renal co-morbidity of level $\mathrm{ASA}=3$ but again not necessarily if he has a respiratory co-morbidity of level $\mathrm{ASA}=2$, etc.

Perhaps not surprisingly, we found that in practice, pre-operative health assessors faced considerable difficulties in using the guidelines. The important number of factors to take into consideration in order to find the correct table and then the specific case within this table, combined with the significant number of tables meant that too much time was being spent by pre-operative health assessors trying to refer to the correct case. In addition, the pre-operative health assessors would need to be able to categorise (i) the type of co-morbidities (ii) their severity (e.g. for determining the patient's ASA grade) (iii) the type of surgical procedures and (iv) their surgical risk grades: all of these steps being necessary before being able to refer to the correct table. All of these tasks are obviously highly knowledge intensive as well as being intellectually demanding. In addition, pre-operative health assessors typically see dozens of different patients a day, each with a wide variety of health conditions and scheduled for various types of surgical procedures. In practice, the consequences are that, if in doubt, pre-operative investigations would probably be requested regardless of the guidelines (i.e. better safe than sorry), thus defeating the purpose of the guidelines in efficiently managing the allocation of pre-operative investigations within care delivery.

\subsection{Pre-operative Tests Recommendations}

We combined the use of an ontology and reasoner in the pre-operative decision support system in order to automatically make recommendations regarding the suitability of tests based on the NICE guidelines. The first step consisted into transforming the NICE tables into rules. This enabled to considerably reduce overlap and redundant information in the current format of the guidelines. The 1242 different possible cases currently covered by the NICE guidelines were reduced to around a hundred rules [20] (see Figure 33). Effectively we modelled instances of the NICE test recommendations as super-test entities in the 


\begin{tabular}{|c|c|c|c|c|}
\hline $\begin{array}{c}\text { Pre-operative } \\
\text { TEST }\end{array}$ & $\begin{array}{l}\text { RECOMMENDED } \\
\text { TEST ENTITY }\end{array}$ & $\begin{array}{c}\text { CONSIDER } \\
\text { TEST ENTITY }\end{array}$ & $\begin{array}{c}\text { NOT RECOMMENDED } \\
\text { TEST ENTITY }\end{array}$ & $\begin{array}{r}\text { TOTAL TEST } \\
\text { ENTITIES }\end{array}$ \\
\hline Blood gases & $\boldsymbol{x}$ & 03 & 01 & 4 \\
\hline Chest X-ray & $\mathbf{O}_{2}$ & $\sim 10$ & 07 & 19 \\
\hline$E C G$ & 09 & 09 & 04 & 22 \\
\hline Full blood count & O5 & O 5 & $O_{2}$ & 12 \\
\hline Haemostasis & $\boldsymbol{x}$ & O6 6 & 03 & 9 \\
\hline Lung function & $\boldsymbol{*}$ & 03 & 03 & 6 \\
\hline Random glucose & $\boldsymbol{x}$ & 06 & 03 & 9 \\
\hline Renal function & 09 & 06 & $O_{2}$ & 17 \\
\hline Urine analysis & * & 23 & 01 & 4 \\
\hline $\begin{array}{c}\text { TOTAL } \\
\text { RECOMMENDATIONS }\end{array}$ & 25 & 51 & 26 & 102 \\
\hline
\end{tabular}

Fig. 3. Categories of OWL Pre-operative test entities according to: (i) pre-operative tests and (ii) type of recommendations

ontology. Definition of these test entities are based: on the 3 dimensions of recommendations (recommended, not recommended, consider) and the 9 categories of pre-operative tests. Patient classification entities are then categorised as subclass entities of pre-operative test entities based on individual medical histories, a process which is described and illustrated in more details in the next section using a practical example. In Figure 3, certain tests have no recommendation test entities associated with them as the guidelines simply do not make explicit recommendations for these tests (but suggest instead that it may appropriate to consider the tests in a specific medical context, as described in Figure 21). Certain tests, such as chest X-ray, ECG, and renal function, are modelled using a larger number of test entities because their medical allocations is dependant on various specific combinations of the 5 input features described previously in 5.1. Others such as blood gases and urine analysis are more generic (e.g. the latter being potentially considered for all adults, regardless of surgery or morbidity). An important modelling consideration was that the test entities were not modelled in order to minimise the number of entities but rather to facilitate the interpretation of the rules by health professionals, as is illustrated in the next section.

\subsection{Example of Pre-operative Tests Reasoning}

Figure 5 provides an example of pre-operative test recommendation based on reasoning on the decision support ontology. The patient: (i) is between 40 and 60 years old, (ii) has unspecified angina pectoris of severity level (iii) ASA $=3$ and is scheduled for a repair of mitral valve procedure (OPCS code K2580). The procedure K2580 is classified in the ontology as a cardio-vascular surgery of risk grade IV. Reasoning on the decision support ontology issues 16 test recommendations (some of which relating to duplicate tests) in total: 7 recommended tests 


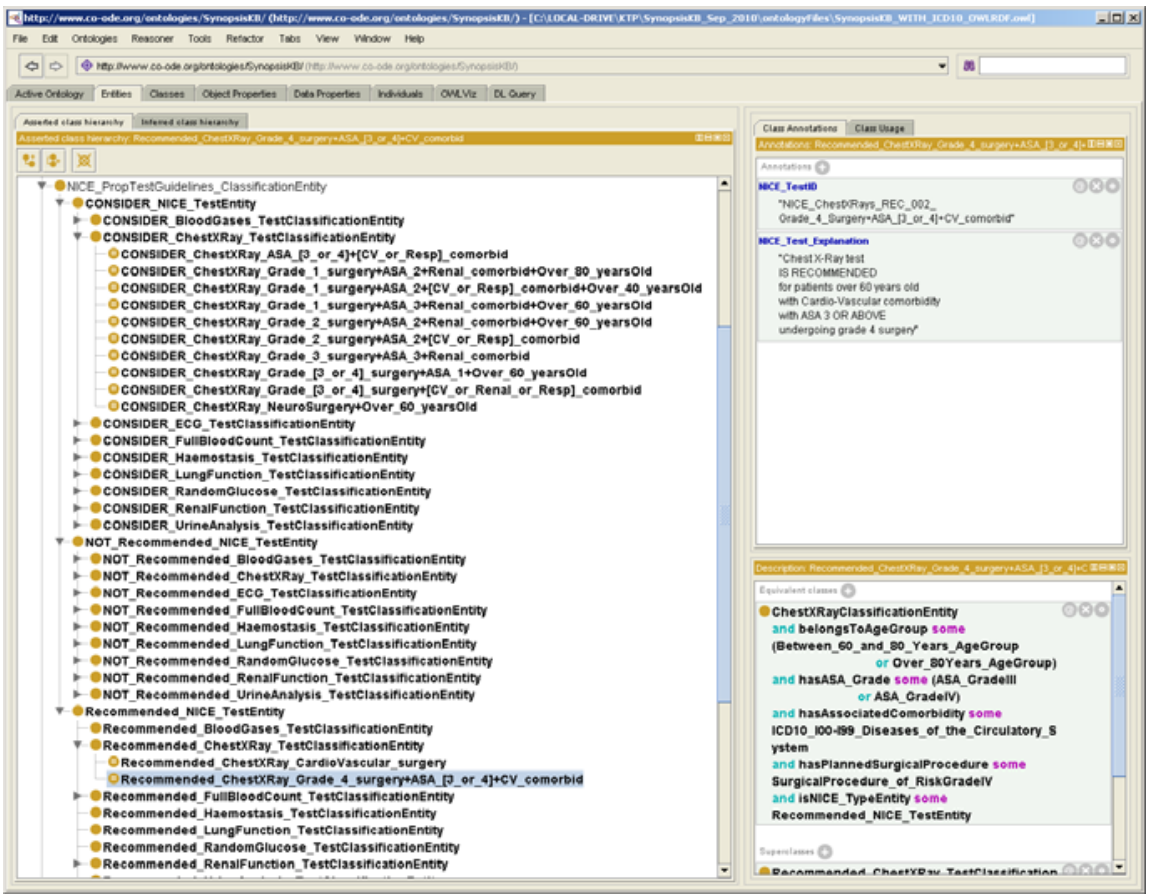

Fig. 4. The NICE guidelines as OWL entity rules as viewed through the Protégé-OWL User Interface

and 9 consider tests. The recommendations are made by the system based on the following reasons:

Chest X-Ray, test recommended: 1 recommended (patient is scheduled for cardio-vascular surgery) +2 consider tests (\{grade $I V$ surgery and cardiovascular co-morbidity $\}$ and $\{A S A=3$ and cardio-vascular co-morbidity $\})$.

ECG, test recommended: 2 recommended (\{cardio-vascular surgery $\}$ and \{cardio-vascular co-morbidity\})

Full Blood Count, test recommended: 1 recommended (cardio-vascular surgery).

Renal function, test recommended: 4 recommended (\{ cardio-vascular surgery $\}$ and $\{A S A=3$ and cardio-vascular co-morbidity $\}$ and $\{$ grade $I V$ surgery $\}$ and last 2 combined )

Haemostasis, consider test 2 consider (\{grade $I V$ surgery $\}$ and $\{$ cardiovascular surgery\})

Blood Gases, consider test 2 consider $(\{A S A=3\}$ and $\{$ grade $I V$ surgery $\})$ Urine Analysis, consider test 1 consider ( all adults over 16 years old)

Random Glucose, consider test 2 consider ( $\{$ grade $I V$ surgery $\}$ and $\{$ cardiovascular surgery\}) 


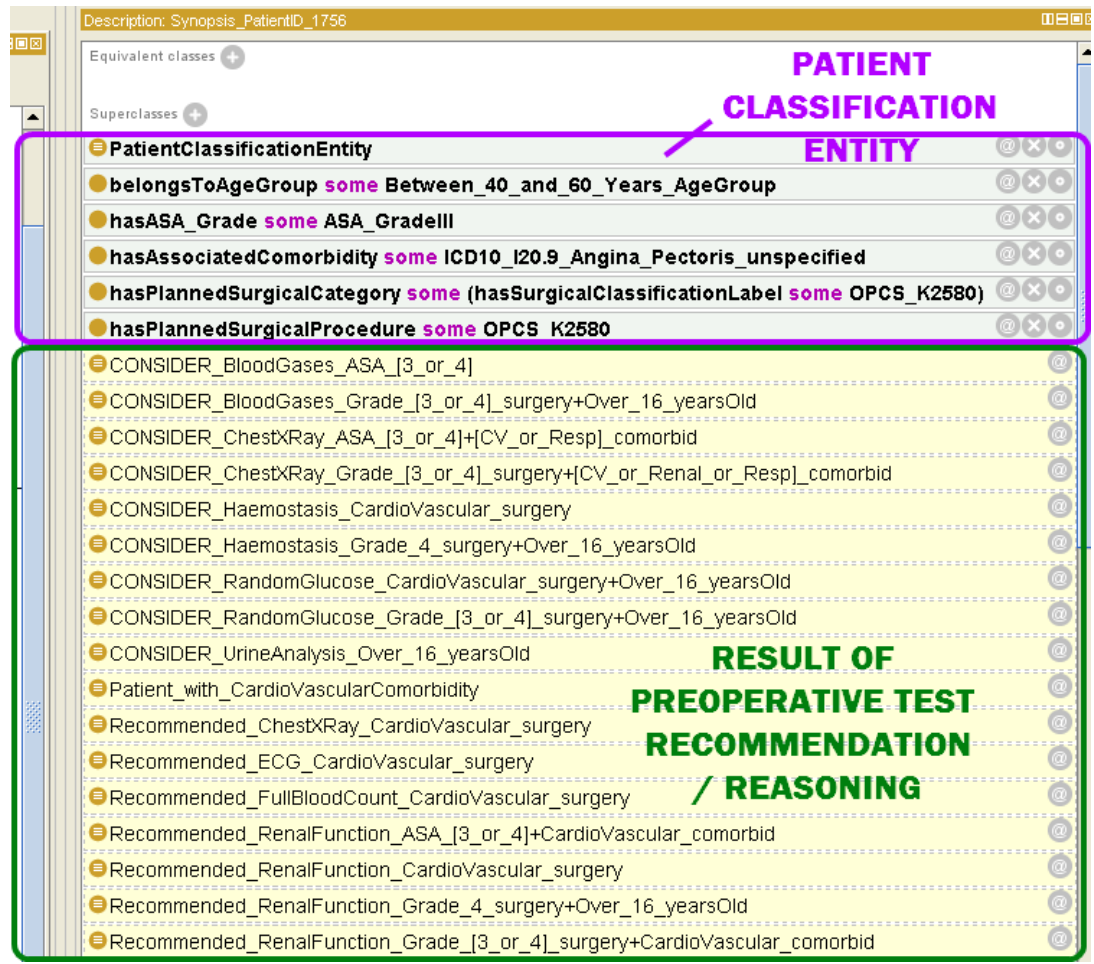

Fig. 5. NICE Pre-operative test recommendations based on reasoning on the decision support ontology

\subsection{Pre-operative Tests and Multiple Morbidities}

A significant development in current world health trends is the substantial increase in the prevalence of chronic diseases and multiple morbidities within the general population, both in developed and developing countries [27. The NICE guidelines do not explicitly deal with the issue of multiple co-morbidities and this is an other aspect in which the system can provide additional decision support functionalities. In the case of a patient with multiple co-morbidities, a test may be recommended for multiple reasons, as illustrated in the previous example. In this case, the system can issue a strong recommendation alongside relevant explanations. Again, the recommendations in the guideline are not mutually exclusive, particularly not in the case of multiple co-morbidities. Thus, we implemented the system so one instance of a "recommended test" within a batch of test results would lead to a positive test recommendation regardless of all the other test recommendations. According to the same principle, if the system returns "consider test" instances along instances of "test not recommended", then the system issues a final "consider test" recommendation. Finally, the system issues a "test not recommended" advice only if all instances retrieved are negative for the specific test. We are hoping to introduce more sophisticated support 
for dealing with multiple conditions as clinical models of complex morbidities are being developed and guidelines become routinely available within the health services 2829130.

\section{Conclusion and Future Work}

We have presented our work to date on the design and implementation of a knowledge-based pre-operative assessment support system. We have discussed how pre-operative assessment is a generic clinical screening process, which purpose is to identify potential risks of complications prior to surgery. By its very nature, the clinical knowledge relevant to pre-operative assessment is potentially limitless. We have proposed some solutions to efficiently harness and manage pre-operative assessment clinical knowledge. The system was developed using semantic web technology including modular ontologies developed in OWL, the OWL API and an automated logic reasoner. This design has provided substantial improvements on earlier versions of the systems, including the ability to tailor patient information collection according to individual medical context, the ability to efficiently manage a vast repository of pre-operative assessment domain knowledge, including classification of surgical procedures and morbidities, and guidelines for routine pre-operative tests. An important modelling consideration was that test entities modelled in the system were not modelled in order to minimise the number of entities but rather to facilitate and optimise the interpretation of rules by health professionals. Future work will involve evaluating the use of the system in clinical settings, in particular with regards standardisation of pre-operative processes and compliances with the guideline recommendations suggested by the system.

Acknowledgement. Dr. Bouamrane is a Scotland Chief Scientist Office (CSO) funded postdoctoral fellow in health informatics and health services research working on the development of information systems in order to facilitate the integration of pre-operative assessment across primary and secondary care in Scotland.

\section{References}

1. Reason, J.: Human error: models and management. British Medical Journal, BMJ 320, 768-770 (2000)

2. Johnston, M.E., Langton, K.B., Haynes, R.B., Mathieu, A.: Effects of Computerbased Clinical Decision Support Systems on Clinician Performance and Patient Outcome: a Critical Appraisal of Research. Annals of Internal Medicine 120(2), 135-142 (1994)

3. Hunt, D.L., Haynes, R.B., Hanna, S.E., Smith, K.: Effects of Computer-Based Clinical Decision Support Systems on Physician Performance and Patient Outcomes A Systematic Review. Journal of American Medical Association, JAMA 280(15), 1339-1346 (1998) 
4. Kaplan, B.: Evaluating informatics applications - clinical decision support systems literature review. International Journal of Medical Informatics 64(1), 15-37 (2001)

5. Bates, D.W., Cohen, M., Leape, L.L., Overhage, J.M., Shabot, M.M., Sheridan, T.: Reducing the Frequency of Errors in Medicine Using Information Technology. Journal of American Medical Informatics Association, JAMIA 8, 299-308 (2001)

6. Sim, I., Gorman, P., Greenes, R.A., Haynes, R.B., Kaplan, B., Lehmann, H., Tang, P.C.: Clinical Decision Support Systems for the Practice of Evidence-based Medicine. Journal of American Medical Informatics Association, JAMIA 8(6), 527 534 (2001)

7. Morris, A.H.: Decision support and safety of clinical environments. Quality and Safety in Health Care 11, 69-75 (2002)

8. Garg, A.X., Adhikari, N.K.J., McDonald, H., Rosas-Arellano, M.P., Devereaux, P.J., Beyene, J., Sam, J., Haynes, R.B.: Effects of Computerized Clinical Decision Support Systems on Practitioner Performance and Patient Outcomes. Journal of American Medical Association, JAMA 293(10), 1223-1238 (2005)

9. Kawamoto, K., Houlihan, C.A., Balas, E.A., Lobach, D.F.: Improving clinical practice using clinical decision support systems: a systematic review of trials to identify features critical to success. British Medical Journal, BMJ 330, 765(8p) (2005)

10. Randell, R., Mitchell, N., Dowding, D., Cullum, N., Thompson, C.: Effects of computerized decision support systems on nursing performance and patient outcomes: a systematic review. Journal of Health Services Research \& Policy 12(14), 242-249 (2007)

11. Taylor, P.: From Patient Data to Medical Knowledge: The Principles and Practice of Health Informatics. BMJ, Blackwell Publishing (2006)

12. García-Miguel, F., Serrano-Aguilar, P., López-Bastida, J.: Pre-operative assessment. The Lancet 362(9397), 1749-1757 (2003)

13. Bouamrane, M.M., Rector, A., Hurrell, M.: Gathering Precise Patient Medical History with an Ontology-driven Adaptive Questionnaire. In: Proceedings of Computer-Based Medical Systems, CBMS 2008, Jyväskylä, Finland, pp. 539-541. IEEE Computer Society Press, Los Alamitos (2008)

14. Bouamrane, M.M., Rector, A., Hurrell, M.: Ontology-Driven Adaptive Medical Information Collection System. In: An, A., Matwin, S., Raś, Z.W., Ślezak, D. (eds.) ISMIS 2008. LNCS (LNAI), vol. 4994, pp. 574-584. Springer, Heidelberg (2008)

15. Bouamrane, M.M., Rector, A., Hurrell, M.: Semi-Automatic Generation of a Patient Pre-operative Knowledge-Base from a Legacy Clinical Database. In: Meersman, R., Dillon, T., Herrero, P. (eds.) OTM 2009. LNCS, vol. 5871, pp. 1224-1237. Springer, Heidelberg (2009)

16. Bouamrane, M.M., Rector, A., Hurrell, M.: Using Ontologies for an Intelligent Patient Modelling, Adaptation and Management System. In: Meersman, R., Tari, Z. (eds.) OTM 2008, Part II. LNCS, vol. 5332, pp. 1458-1470. Springer, Heidelberg (2008)

17. Rector, A.: Modularisation of domain ontologies implemented in description logics and related formalisms including OWL. In: Proceedings of the 2nd International Conference on Knowledge Capture, K-CAP 2003, Sanibel Island, FL, USA, October 23-25, pp. 121-128. ACM, New York (2003)

18. Rector, A., Horridge, M., Iannone, L., Drummond, N.: Use Cases for Building OWL Ontologies as Modules: Localizing, Ontology and Programming Interfaces \& Extensions. In: Proceedings of 4th Int Workshop on Semantic Web Enabled Software Engineering, SWESE 2008, with ISWC 2008, Karlsruhe, Germany (October 2008) 
19. Bouamrane, M.M., Rector, A., Hurrell, M.: Development of an Ontology of Preoperative Risk Assessment for a Clinical Decision Support System. In: Proceedings of the 22nd IEEE International Symposium on Computer-Based Medical Systems, CBMS 2009, 4th Special Track on Ontologies for Biomedical Systems, Albuquerque, USA. IEEE Computer Society Press, Los Alamitos (August 2009)

20. Bouamrane, M.M., Rector, A., Hurrell, M.: A Hybrid Architecture for a Preoperative Decision Support System using a Rule Engine and a Reasoner on a Clinical Ontology. In: Polleres, A. (ed.) RR 2009. LNCS, vol. 5837, pp. 242-253. Springer, Heidelberg (2009)

21. Knublauch, H., Fergerson, R.W., Noy, N.F., Musen, M.A.: The Protégé OWL Plugin: an open development environment for Semantic Web applications. In: McIlraith, S.A., Plexousakis, D., van Harmelen, F. (eds.) ISWC 2004. LNCS, vol. 3298, pp. 229-243. Springer, Heidelberg (2004)

22. OWL 2.: Web Ontology Language document overview (2009), http://www.w3.org/tr/owl2-overview/

23. Horridge, M., Bechhofer, S., Noppens, O.: Igniting the OWL 1.1 touch paper: the OWL API. In: Proceedings of the Third International Workshop of OWL Experiences and Directions, OWLED 2007, Innsbruck, Austria (2007)

24. Sirin, E., Parsia, B., Grau, B.C., Kalyanpur, A., Katz, Y.: Pellet: A practical OWLDL reasoner. Journal of Web Semantic 5(2), 51-53 (2007)

25. Palda, V.A., Detsky, A.S.: Perioperative assessment and management of risk from coronary artery disease. Annals of internal medicine 127(4), 313-328 (1997)

26. Munro, J., Booth, A., Nicholl, J.: Routine pre-operative testing: a systematic review of the evidence. Health Technology Assessment 1(12), 76 (1997)

27. W.H.O: Preventing chronic diseases: a vital investment. World Health Organization, Geneva. Technical report (2005)

28. Fortin, M., Dubois, M.F., Hudon, C., Soubhi, H., Almirall, J.: Multimorbidity and quality of life a closer look. Health and Quality of Life Outcomes 5(52), 8 (2007)

29. Mercer, S.W., Smith, S.M., Wyke, S., O’Dowd, T., Watt, G.C.: Multimorbidity in primary care: developing the research agenda. Journal of Family Practice 26(2), 79-80 (2009)

30. Valderas, J.M., Starfield, B., Sibbald, B., Salisbury, C., Roland, M.: Defining comorbidity: Implications for understanding health and health services. Annals of Family Medicine 7(4), 357-363 (2009) 